\title{
Detailed expression profile of all six Glypicans and their modifying enzyme Notum during chick embryogenesis and their role in dorsal-ventral patterning of the neural tube
}

\section{Article}

Accepted Version

Creative Commons: Attribution-Noncommercial-No Derivative Works 4.0

Saad, K., Otto, A., Theis, S., Kennerley, N., Munsterberg, A., Luke, G. and Patel, K. (2017) Detailed expression profile of all six Glypicans and their modifying enzyme Notum during chick embryogenesis and their role in dorsal-ventral patterning of the neural tube. Gene, 609. pp. 38-51. ISSN 0378-1119 doi: https://doi.org/10.1016/j.gene.2017.01.032 Available at https://centaur.reading.ac.uk/68863/

It is advisable to refer to the publisher's version if you intend to cite from the work. See Guidance on citing.

To link to this article DOI: http://dx.doi.org/10.1016/j.gene.2017.01.032

Publisher: Elsevier

All outputs in CentAUR are protected by Intellectual Property Rights law, including copyright law. Copyright and IPR is retained by the creators or other copyright holders. Terms and conditions for use of this material are defined in the End User Agreement. 


\section{www.reading.ac.uk/centaur}

\section{CentAUR}

Central Archive at the University of Reading

Reading's research outputs online 
Title

Detailed expression profile of all six Glypicans and their modifying enzyme Notum during chick embryogenesis and their role in dorsal-ventral patterning of the neural tube

\section{Authors}

Kawakeb Saad ${ }^{1}$, Anthony Otto ${ }^{1}$, Susanne Theis ${ }^{1}$, Niki Kennerley², Andrea

Munsterberg ${ }^{2}$, Graham Luke ${ }^{1}$, Ketan Patel ${ }^{1,3, *}$

Address

1 School of Biological Sciences, University of Reading, Reading, UK

2 School of Biological Sciences, Norwich Research Park, University of East Anglia, Norwich, NR4 7TJ

3 Freiburg Institute for Advanced Studies (FRIAS), University of Freiburg, Freiburg, Germany

Corresponding author ketan.patel@reading.ac.uk

\section{Address}

School Biological Sciences

University of Reading

Hopkins Building

Reading

RG6 6UB, UK

Key Words

Embryo, Chick, signalling, Shh, BMP, FGF, Glypican, Notum 


\begin{abstract}
Vertebrate development is orchestrated by secreted signalling molecules that regulate cell behaviour and cell fate decisions during early embryogenesis. The activity of key signalling molecules including members of Hedgehog, Bone Morphogenetic Proteins and Wnt families are regulated by Glypicans, a family of GPI linked polypeptides. Glypicans either promote or inhibit the action of signalling molecules and add a layer of complexity that needs to be understood in order to fully decipher the processes that regulate early vertebrate development. Here we present a detailed expression profile of all six Glypicans and their modifying enzyme Notum during chick embryogenesis. Our results strongly suggest that these proteins have many as yet undiscovered roles to play during early embryogenesis. Finally, we have taken an experimental approach to investigate their role during the patterning of a key embryonic structure - the neural tube. In particular, we show that overexpression of Notum leads to the dorsalisation of this structure.
\end{abstract}

\title{
Introduction
}

The development of multicellular organisms is regulated by signalling pathways activated by a diverse range of secreted molecules including wingless (Wnt), Hedgehog (Hh), Bone Morphogenetic Protein (Bmp) and Fibroblast Growth Factor (Fgf) family members. A simplistic view of their action posited that these proteins diffused until they interacted with their receptor to initiate an intracellular signalling cascade, usually culminating in a change of gene expression in the target cell, which then influences cell fate and behaviour. 
In the last few years, focus has turned on Heparan Sulfate Proteoglycans (HSPGs) as many genetic studies have demonstrated that they play a central role in regulating signalling during development. HSPGs are abundant cell-surface glycoproteins, which act as co-receptors in signalling processes (Bernfield et al., 1999) and contain one or more covalently attached heparan sulfate (HS) chains (Esko et al., 2009). HSPGs are classified into several families based on their core protein structure. Syndecans, Perlecan and Glypicans (Gpc) are examples of major cell surface HSPGs. Syndecans are transmembrane proteoglycans that contain a highly conserved carboxy-terminal cytoplasmic domain. The HS chains attach to serine residues distal to the plasma membrane. Pelecans are secreted extracellular matrix (ECM) proteoglycans that are not cell-membrane linked. In contrast, Gpcs are attached to the cell surface by a Glycosylphosphatidylinositol (GPI) anchor (Lin, 2004). Gpc core proteins are $60-70 \mathrm{kDa}$ in size and share common structural features across the family. Each Gpc can be divided into three structural domains; the linker domain at the C-terminal end connects the core protein to a GPI anchor in the cell membrane, adjacent to the linker region are the attachment sites for glycosaminoglycan (GAG) chains. The insertion sites are within 50 amino acid residues of the membrane anchor, positioning the GAG chains close to the cell membrane. The third Gpc domain is a globular cysteine-rich domain (CRD) (Pei and Grishin, 2012) .

The tertiary structure of the CRD is thought to remain constant between Gpc family members due to the presence of 14 highly conserved cysteine residues that are predicted to form stabilizing disulphide bonds. Gpc GAG chains are linear sugar polymers consisting of a repeating disaccharide unit. The GAG chains of Gpcs carry a negative charge, allowing promiscuous interaction with basic charged growth 
factors (Filmus and Capurro, 2008). Pertinent to the Gpc function is the enzyme Notum since it is able to cleave the former near the cell membrane (Kreuger et al., 2004).

Gpcs have received much attention as they have been shown to modulate the activity of the major classes of secreted proteins that control the development of all animals. The discoveries that mutations in Gpc genes result in diseases such as Simpson-Golabi-Behmel syndrome (Pilia et al., 1996), Omodysplasia (CamposXavier et al., 2009) and cancer has brought them into focus (Filmus and Selleck, 2001).

The Gpc gene family members have been conserved during animal evolution and are found in both invertebrates and vertebrates (Filmus et al., 2008; Filmus and Selleck, 2001). There are two Gpc genes in Drosophila: Division abnormally delayed (Dally) and Dally-like protein (Dlp) (Khare and Baumgartner, 2000; Khare et al., 2000), whereas six Gpcs have been identified in mammals (Gpc1 to Gpc6) (Song and Filmus, 2002).

Based on amino acid homology, mammalian Gpcs can be subdivided in two distinct groups. The first group including Gpc1, Gpc2, Gpc4 and Gpc6 with 35\%-63\% sequence similarity; the second include Gpc3 and Gpc5, which show 54\% similarity (Veugelers et al., 1999), whereas the homology between of the two groups is only $17 \%-25 \%$. The role of Gpcs in modulating signalling activity of secreted proteins is context dependent. In some cases Gpcs can promote the activity of the signalling molecule; for example Gpc3 binds to Wnt proteins and the Wnt receptor Frizzled, to either facilitate or stabilise the complex (Capurro et al., 2014). However, in other situations they attenuate signalling activity; for example Gpc3 inhibits Shh signalling by promoting ligand endocytosis (Capurro et al., 2008). 
A number of studies have documented the expression of Gpc or Notum during vertebrate development (Luxardi et al., 2007; Niu et al., 1996; Ybot-Gonzalez et al., 2005), however to our knowledge none have reported a comprehensive profile of all Gpcs and Notum over the entire period of early embryogenesis. Here we provide a detailed expression profile of all Gpcs and Notum during chick embryogenesis starting at the stage of axial mesoderm formation to late limb bud stages $(\mathrm{HH}-4$ to $\mathrm{HH}-26)$ (Hamburger and Hamilton, 1951). We show that each gene has its unique expression profile that is temporally highly dynamic. Some Gpcs show strong expression in multiple sites whereas others are more restricted. We show that many are expressed at key patterning sites including Hensen's Node, the notochord, and the floor and roof plates of the neural tube. Finally, we show an important role for Gpcs during the dorsal ventral patterning of the neural tube through the overexpression of Notum, which culminated in a dramatic dorsalisation of key cell determinant markers.

\section{Methods}

\section{Cloning of chick Glypican and Notum genes}

cDNA was prepared from RNA extracted from whole $\mathrm{HH}-25$ chick embryos. RT-PCR was performed using the following gene specific primers.

cGPC1 F5'-3'GCGAATCTGTCCGCAAGGCTACAC,

R 3'-5'CTAAGCCGTCCCCCATCACTTCAG amplification product size of 1136bp.

cGPC2 F5'-3'GGCAAAAGAAGCAGCAGAGCCTGTTAAAG,

R3'-5'TCATCACCAGGTCTCCATCACACAGC amplification product size of 828bp. cGPC3 F5'-3'CTGCTCGAGGAGGATGGAGGAGAAGTAC, 
R3'-5'CTGTACCTCTCCACGACTTCTTGCCC amplification product size of 1083bp. cGPC4 F5'-3'GCGACCACTTGAAAGTCTGCTCACAAG,

R3'-5'GCTGCTTGTGATAAACCGCTACTGGG amplification product size of 1400bp. cGPC5 F5'-3'GAAAGTTTTCCAGCTGCGTCAGCTCG,

R3'-5'GGCAAGGGTTTCTTCGCTGTCTCTTG amplification product size of 1042bp. cGPC6 F5'-3'TTCTTGCAATTCCAGGGGAACATTTGAG,

R3'-5'ATCCAAACTTGTGCCAGCAGCAGTTG amplification product size of 1001bp. cNotum F5'-3' ATGCCTTCATGGGAGCGCTGATC,

R3'-5' AACTGGTCCCTGATAGTGGGGCACG amplification product size of 768bp PCR products were cloned into pDRIVE vector.

\section{Preparation of embryos}

All experiments were performed on Gallus gallus domesticus chicken embryos. Fertilised eggs were purchased from Henry Stewart and Co, UK. Eggs were incubated at $38^{\circ} \mathrm{C}$ and $80 \%$ humidity.

\section{Whole mount in-situ hybridisation}

Whole mount in-situ hybridisation was performed according to Nieto (Nieto et al., 1996). A minimum of 5 embryos were processed for each experimental outcome reported here.

\section{Cryosectioning}

Embryos were washed 3 times with PBS for 15 minutes to remove fixative and equilibrated in 5,15 and $30 \%(\mathrm{w} / \mathrm{v})$ sucrose in PBS overnight at $4^{\circ} \mathrm{C}$ before freezing in Optimum Cutting Temperature embedding media (O.C.T. Leica Microsystems). 30 $\mu \mathrm{m}$ sections were cut using a cryostat (Bright instruments UK). 


\section{Photography}

Whole mount embryos were photographed using a Nikon CoolPix camera mounted on a Nikon dissecting microscope. Processed cryo-sections were photographed using an Axiocam digital camera fitted on a Zeiss Axioscope fluorescent microscope connected with Zeiss Axiovision computer software version 3. Images were processed using Adobe Photoshop Elements 6.

\section{Electroporation}

Mouse Notum (mNotum) clone was gift from Jorge Filmus (University of Toronto Canada) in pTREACER-FEV5-HisAvector. The mNotum insert was excised with Ncol and Xbal restriction enzymes, and then cloned to the pSLAX vector. Notum insert was cut out from pSLAX with Notl and Xbal then cloned into pCAB5 vector (designed by Jon Gilthore and constructed by Allison Hunter - MRC Centre for Developmental Neurobiology, UK). It contains the beta-actin promoter and an IRES to express GFP from the same backbone.

Eggs were incubated for 2 days to reach $\mathrm{HH}$ stage 10-12. Electroporation mixture was made of Carboxymethyl cellulose (CMC), $1 \%$ fast green in PBS. The electroporation mixture was prepared by dissolving $0.5 \mathrm{~g}$ of $\mathrm{CMC}$ in $25 \mathrm{ml}$ water and then adding $15 \mathrm{ml}$ of 10XPBS. Once the CMC was completely dissolved, $105 \mu \mathrm{l}$ of $100 \mathrm{mM} \mathrm{MgCl}$ was added. The solution was aliquoted in $400 \mu \mathrm{l}$ volumes. Thereafter, $75 \mu \mathrm{l}$ of Fast Green was added to each aliquot. $2 \mu \mathrm{g}$ of DNA was added to the electroporation mixture in ratio of 1:2. A capillary needle was loaded with prepared DNA/Fast Green mix, which was injected into lumen of the neural tube until the dye filled the entire space using Eppendorf Femtojet Express Microinjector (Eppendorf, UK). PBS solution containing penicillin-streptomycin was dropped on the newly injected manipulation site. Electrodes were positioned to the left and right of the 
embryo. Four pulses of 30 milli-seconds at 15 Volts were applied using an Intracel TSS 20 electroporater (Micro Control Instruments, UK) to enable DNA transfection into one half of the neural tube. Eggs were sealed and re-incubated at $37^{\circ} \mathrm{C}$ and $80 \%$ humidity for $16-24$ hours. Surviving embryos were investigated for GFP expression using Zeiss Axioscope fluorescent microscope and an Axiocam digital camera. Embryos showing GFP expression were fixed in 4\% PFA/PBT and stored at $4^{\circ} \mathrm{C}$ for further analysis.

\section{Fluorescence microscopy and image analysis}

Sections stained with fluorescent secondary antibodies were analysed using a Leica DM4000B fluorescent microscope. Pictures were taken using a DC500 camera system and JPEG formatted images were overlaid if necessary using Leica image analysis software. Minor adjustments to brightness and contrast were made using Adobe Photoshop 6 elements.

\section{Statistical analysis}

Data were processed for statistical significance using independent samples t-test at the $95 \%$ confidence interval. All data are presented as means and standard errors of the mean (S.E.M).

\section{Results}

\section{Expression of GPC1-6 at HH-4}

In order to understand Glypicans (abbreviated henceforth to Gpc) and Notum function during embryonic development in-situ hybridization was used to detail the expression of these genes during development of the chick embryo.

At stage $\mathrm{HH}-4$, Gpc1 was expressed from the anterior to the posterior of the embryo along the midline (Fig. 1A). The expression was strong in the head process, 
Hensen's node and primitive streak. Transverse section through the head process region (Fig. 1A') showed that Gpc1 was strongly expressed in the ectoderm of the head fold and it became weaker in the ingressing mesoderm (Fig. 1A'"). Transverse section at the level of Hensen's node (Fig. 1A") showed that Gpc1 was expressed strongly in the ectoderm as well as the Node.

Gpc2 expression at $\mathrm{HH}-4$ was found in low levels in the anterior part of the embryo (Fig. 1B). Transverse section through the Hensen's node (Fig, 1B') showed that Gpc2 was weakly expressed in the epiblast and mesoderm. Transverse section though the primitive streak (Fig. 1B") showed that Gpc2 was expressed in the ingressing cells in the primitive streak but to a lesser level in tissue that had completed this process.

Gpc3 was expressed in the head process region, Hensen's node, primitive streak at HH-4 (Fig. 1C). Transverse section in the area of head process (Fig. 1C') demonstrated strong expression in the ectoderm. Transverse sections at the level of Hensen's node (Fig. 1C") showed low level Gpc3 expression in the ingressing cells. Gpc4 was expressed strongly in the anterior and posterior most parts of the embryo (Fig. 1D). At Hensen's node (Fig. 1D') Gpc4 was expressed in the epiblast and the ingressing cells. In the primitive streak Gpc4 was strongly expressed in the ingressing mesoderm (Fig. 1D"). Gpc5 was expressed at $\mathrm{HH}-4$ along the primitive streak, Hensen's node and weakly at the anterior of the primitive streak. The expression extended from the primitive streak to the area pellucida (Fig. 1E). Interestingly, its expression in the ectoderm and epiblast was punctate (Fig. 1E'-E'”). Gpc6 displayed very low level expression (Fig. 1F) but was most prominent at the epiblast immediately adjacent to Hensen's Node (Fig. 1F'). 
In summary at $\mathrm{HH}-4, G p c 1$, Gpc4 and Gpc5 were expressed widely and robustly contrasting Gpc2, Gpc3 and Gpc6 which were found at low levels.

\section{Expression of Gpc1-6 at HH 7-8}

At HH-7 Gpc1 was strongly expressed in the head fold, head process, Hensen's node and primitive streak (Fig. 2A). Most striking was the segmental pattern immediately anterior to Hensen's Node. Gpc1 expression was at low levels in the prechordal plate mesoderm. Gpc1 was expressed strongly in the neural plate (Fig. $2 A^{\prime}$ ), and primitive streak (Fig. 2A") both in the epiblast and ingressing cells.

At $\mathrm{HH} 7+$, Gpc2 showed limited expression at $\mathrm{HH}-7-$ at the anterior part of the embryo, in the head process region and in Hensen's Node (Fig. 2B and 2B'). Gpc3 was strongly expressed in the anterior part of the embryo and the head fold at $\mathrm{HH}-7$ (Fig. 2C). Transverse section in the head fold region (Fig. 2C') showed Gpc3 expressed in the neural tube. Gpc3 expression in the epiblast at the level of Hensen's Node was particularly strong (Fig. 2C").

Gpc4 was expressed strongly in the head fold, somites, Hensen's Node and primitive streak at $\mathrm{HH}-8$. Future head region showed strong expression in the neural plate and head ectoderm (Fig. 2D').

At HH-7 Gpc5 was expressed widely in the developing embryo. Expression was high in the head fold, Hensen's node and primitive streak. At the level of Hensen's Node it was found in the epiblast and mesoderm (Fig. 2E").

At $\mathrm{HH}-7$ Gpc6 was expressed at low levels in the head process (Fig. 2F). Low levels of Gpc6 expression were detected in the neural tube (Fig. 2F'). At the level of Hensen's node Gpc6 was expressed in the epiblast (Fig. 2F"). 


\section{The expression of Gpc1-6 at HH-12}

Gpc1 at $\mathrm{HH}-12$ showed robust expression especially in the anterior and posterior of the gastrulated embryo (Fig. 3A). In the anterior portion of the embryo it was expressed the head mesenchyme, hindbrain and otic placode (Fig. 3A'). Gpc1 also found in keel of the pharynx (Fig. 3.A'). More posteriorly it was expressed the neural tube, notochord and dermomyotome (Fig. 3A"). Gpc1 was however stronger in the somites and notochord at the posterior of the embryo (Fig. 3A"'). Low levels of the gene were detected in the intermediate and lateral plate mesoderm.

Similar to Gpc1, Gpc2 at the same stage was expressed strongly in the anterior of the embryo especially the fore, mid and hind brain (Fig. 3B). Also Gpc2 was expressed in the newly formed somites. Transverse section at the level of the forebrain showed expression of Gpc2 in neural tube and head mesenchyme (Fig. $\left.3 B^{\prime}\right)$. Transverse section at the level of the hindbrain indicated that Gpc2 was expressed in the neural plate and head mesenchyme. Also it was expressed strongly in the ectodermal floor of the pharynx (Fig. 3B"). Transverse section at the level of the first differentiated somite (Fig. 3B'") showed that Gpc2 expression was found in sclerotome and lateral plate mesoderm.

Expression of Gpc3 at $\mathrm{HH}-12$ was restricted to the anterior part of the developing embryo (Fig. 3C). Gpc3 was expressed in the mid and hindbrain (Fig. 3C) and the anterior tip of foregut (Fig. 3C').

Gpc4 was widely expressed in the developing embryo at $\mathrm{HH}-11$ with striking expression in the fore, mid and hindbrain, neural tube and both undifferentiated and differentiated somites (Fig. 3D). At the level of differentiated somites, it was expressed both in the dermomyotome and sclerotome. These views also revealed expression in the intermediate and lateral plate mesoderm (Fig. 3D'”). Gpc4 was 
also expressed in the roof plate and floor plate and at low levels in the notochord (Fig. 3D"').

At $\mathrm{HH}$ 10-11 Gpc5 was expressed throughout the whole embryo and especially strongly in the head region, somites and primitive streak (Fig. 3E). Transverse section in the head region showed that Gpc5 was strongly expressed in the neural tube (Fig. 3E'); with weaker expression in the head mesenchyme. Furthermore Gpc5 was heavily expressed in the keel of the pharynx. Transverse section at the level of differentiated somites showed that Gpc5 was expressed in the neural tube (Fig. 3E'). Also some faint expression was found in the splanchnic lateral plate mesoderm. Gpc5 was expressed in the somites (Fig. 3E'”).

Gpc6 expression at $\mathrm{HH}-12$ was restricted to the all three compartments of the developing brain (Fig. 3F and F').

In summary at stage $\mathrm{HH} 10-12$ Gpc1, Gpc4 and Gpc5 expression was prominent in neural tube and somites. On the other hand Gpc2 and Gpc3 were restricted to only the anterior part of the chick embryo.

\section{Expression of Gpc1-6 at HH 18-19}

By $\mathrm{HH}-19$ Gpc1 was expressed in head region, the fore, mid, and hind-brain (Fig. 4A). Gpc1 was expressed in fore and hind limb buds and was strongly expressed in the Apical Ectodermal Ridge (AER) of the hind limb. Gpc1 was found in the first and second pharyngeal branches. Transverse section at the level of cranial somites (Fig. 4A') showed that Gpc1 was expressed in the neural roof plate and the myotome. Transverse section through the forelimb bud region showed that Gpc1 was expressed in the neural roof plate and myotome (Fig. 4A"). Also Gpc1 was 
expressed in the forelimb mesenchyme. In the hind limb (Fig. 4A'") it was expressed in limb bud ectoderm.

By stage HH-19 Gpc2 had become more restricted in the head and occipital region. There was some expression in the anterior intestinal portal (Fig. 4B). Transverse section in the head region shows expression in the lateral side of the lens vesicle and nasal placode (data not shown). Transverse section in the cranial somite (Fig. 4B') showed expression in the neural floor plate and endoderm. At fore limb level (Fig. 4B") expression was found in the floor plate and fore limb bud mesenchyme. Furthermore Gpc2 was expressed in the heart region (Fig. 4B").

By HH 18 Gpc3 was expressed in the anterior part of developing embryo (Fig. 4C) with expression in the forebrain, first and second branchial arches, and anterior intestinal portal. Gpc3 was expressed in the posterior part, the mesoderm of the inter limb region and in fore and hind limb bud. The head region shows Gpc3 expression in the upper part of the neural tube and distal part of the optic cup. At the cranial somite level Gpc3 was expressed in the middle of the neural tube (Fig.4C').

Gpc3 expression was detected in the fore limb bud mesenchyme and splanchnic mesoderm (Fig4 C"). In the hind limb it was expressed in the limb mesenchyme and in the lining of embryonic coelom (Fig. 4C'”').

At $\mathrm{HH}-19$ Gpc4 was widely expressed in the embryo. Fig. 4D shows expression the first, second and third branchial arches, and differentiated as well as epithelial somites. In addition, Gpc4 was expressed in the fore and hind limb buds. Transverse section through the cranial somite showed expression in the neural tube, sclerotome and dermomyotome (Fig.4D'). At the fore limb (Fig.4D") and hind limb level (Fig. 4D"') expression was found in the neural tube, dermomyotome and sclerotome. Gpc4 was expressed strongly and in the intermediate mesoderm. 
Gpc5 expression at $\mathrm{HH}-19$ had become limited to the posterior part of the embryo. The lateral view of the embryo showed that Gpc5 was expressed strongly in the forelimb bud; inter-limb region and hind limb bud (Fig.4E). Transverse section at the forelimb bud level showed expression in the forelimb mesenchyme and weak expression in the neural tube (Fig.4E'). Transverse section through the inter-limb region (Fig. 4E") showed strong expression in the lateral plate mesoderm and more posteriorly in the hind limb bud mesenchyme (Fig. 4E”'). Gpc6 expression at $\mathrm{HH}-19$ was limited to the otic placode (Fig. 4F).

\section{GPC1-6 expression at $\mathrm{HH}-22$}

Lateral view of an embryo at $\mathrm{HH}-22$ showed that Gpc1 was widely expressed at this stage (Fig.5A) including fore, mid, and hindbrain. Expression was also detected in the first, second and third branchial arches and was strongly expressed in the fore and hind limb buds. Transverse section through the cranial somite (Fig. 5A') showed that Gpc1 was expressed in the dermomyotome (Fig. 5A'). Transverse section at the forelimb region showed that Gpc1 expression was localised in both sclerotome and dermomyotome forelimb mesenchyme (Fig. 5A"). Gpc1 expression in the hind limb AER and dorsal limb ectoderm was prominent as well as in the dorsal root ganglia (Fig. 5A"').

At $\mathrm{HH}-22$ Gpc2 was still strongly expressed in the anterior part of the embryo especially in the head and neck (Fig. 5B). Gpc2 was weakly expressed in the posterior part of the embryo, and at low levels in the hind limb and tail bud. Transverse section through the cranial somite (Fig. 5B') showed that Gpc2 expression was in the ventral part of the neural tube adjacent to the floor plate (Fig. 5B'). Transverse section at the level of the forelimb bud showed that Gpc2 
expression was found in the intermediate mesoderm as well as the distinct region adjacent to the floor plate (Fig. 5B" and 5B'”').

Lateral view of a HH-22 embryo (Fig. 5C) showed that Gpc3 was strongly expressed the fore, midbrain and cranial placodes. Transverse section of the cranial somites (Fig. 5C') showed weak Gpc3 expression in the dorsal root ganglia (Fig. 5C').

The neural tube showed expression under the roof plate at the level of the fore limb (Fig. 5C"). There was strong expression in the forelimb bud mesenchyme. Similar expression was found at the hind limb level (Fig. 5C"').

At $\mathrm{HH}-22$ (Fig. 5D) Gpc4 was widely expressed the head region, eye and strongly expressed in the midbrain and hindbrain. Gpc4 expression was found in the branchial arches and in the fore and hind limb buds. Transverse section at the head region showed that Gpc4 was expressed in the head ectoderm. Transverse section at the cranial somite region showed expression in the dorsal neural tube (roof plate) and dermomyotome (Fig. 5D') both of which extended along the posterior axis. In both fore and hind limbs, Gpc4 expression was found only in the dorsal mesenchyme (Fig. 5D"' and D"').

At HH-22 Gpc5 expression was detected at low levels in the fore, inter and hind limb regions (Fig. 5E). Transverse section through the forelimb bud showed that Gpc5 transcription was weakly expressed in the DRG and also in the limb mesenchyme (Fig.5E'). More posteriorly it was expressed in the hind limb bud mesenchyme and sclerotome (Fig.5E'”).

At $\mathrm{HH}$-stage 22 Gpc6 expressions was solely expressed in the otic placode (Fig.5F). 


\section{The expression of Gpc1-6 at $H H$ 24-25}

At $\mathrm{HH}-24$ Gpc1 was expressed in the head region; the frontal nasal process and the branchial arches (Fig. 6A). Gpc1 was found in the cranial somites and expression become stronger in the trunk somites (Fig. 6A).

Gpc2 was strongly expressed in the first branchial arch and in limb buds. It was expressed strongly in the proximal fore and hind limb buds in contrast to its weak expression in the distal part (Fig. 6B).

Gpc3 expression at $\mathrm{HH}-24$ had become exquisitely limited to the mesenchyme adjacent to the dorsal-ventral interface of the limb buds (Fig. 6C).

At $\mathrm{HH}-24$ Gpc4 was expressed widely; in the branchial arches region, and highly expressed in the cranial somites and trunk somites (Fig. 6D). It was strongly expressed in the caudal and tail bud somites. However Gpc4 expression had decreased in the head region. At HH-24 Gpc5 expression became limited and less intense. There was some expression in the frontal nasal process and bronchial arch regions (Fig. 6E). By HH-25 Gpc6 was expressed very weakly except for the otic placode and heart (Fig. 6F).

\section{Notum expression at stage $\mathrm{HH} 4-7$}

Notum was expressed along the entire primitive streak at $\mathrm{HH}-4$ with expression being located predominantly in the epiblast (Fig. 7A-D). By HH-7 (Fig. 7A') Notum was expressed in the head fold region, segmenting paraxial mesoderm as well as the Hensen's node region (Fig. 7A'), Transverse section at the segmenting mesoderm level showed that Notum was expressed in the notochord and mesoderm undergoing somite formation (Fig. 7C'). In contrast it was weakly expressed in the neural tube (Fig. 7B'). Strong Notum expression was detected in ectoderm and in the notochord in the posterior of the post-gastrulated embryo. (Fig. 7D'). 


\section{Notum expression at stage $\mathrm{HH} 10-13$}

At $\mathrm{HH}-10$, Notum was expressed in the fore, mid and hind brain, the somites and lateral plate mesoderm (Fig. 8A). The transcription of Notum was very strong in the notochord and remaining primitive streak (Fig. 8A). Transverse section in the head region showed expression in the neural tube, neural crest cells, floorplate and notochord (Fig. 8B). Transverse section at the level of the epithelial somites showed that expression through the somatic mesoderm, in the neural roof plate and notochord (Fig. 8C). By HH-13 Notum was weakly expressed in the mid and hind brain and first and second pharyngeal arches (Fig.8 A'). In contrast, extremely strong expression was found in the somites, notochord and to a lesser extend in the neural tube. In the somites, expression was confined solely to the dermomyotome (Fig. 8B', C').

\section{Notum expression at stage $\mathrm{HH} 18-26$}

At $\mathrm{HH}-18$ Notum was robustly expressed in all somites, intermediate mesoderm and in the apical ectodermal ridge of the fore and hind-limb buds (Fig. 9A). Expression of Notum was also detected in branchial arches 1-3 (Fig. 9A). Transverse section at the cranial somite level showed Notum expression in the dorsal neural tube, notochord and dermomyotome (Fig. 9B). At fore limb level, expression was detected in the AER as well as underlying mesoderm (Fig. 9C), a situation also found in the hind limb (Fig. 9D) and apical ectoderm ridge.

At $\mathrm{HH}-22$, Notum was expressed in the first branchial arch, and to a lesser extent in the second and third branchial arches. In addition, Notum was strongly expressed in the dorsal somatic region and in the fore and hind limb bud (Fig. 9A'). Transverse sections through the cranial somites showed that Notum was strongly expressed in the dermomyotome and the dorsal root ganglions (Fig. 9B'). Weak Notum expression 
was found in the dorsal neural tube. Transverse section at the level of forelimb bud (Fig. 9.C') showed that Notum was strongly expressed in the dorsal ectoderm and adjacent mesoderm of the fore and hind limb and in the AER (Fig. 9C' and 9D').

By $\mathrm{HH}-26$ Notum was expressed in all somites and branchial arches as well as the limbs (Fig. 9A"). Transvers section in the cranial region showed Notum expression in the dorsal root ganglia (DRG), neural tube and roof plate (Fig. 9B"). At the trunk level, expression was clear in the dermomyotome (Fig. 9C"). Transverse section at the fore limb bud showed Notum expression in the AER and in the dorsal limb mesoderm (Fig. 9D”).

Note a more detailed expression of all Gpcs and Notum in the limbs will be presented in separate study (submitted).

\section{Notum over-expression in the neural tube}

The neural tube is patterned along the $\mathrm{D}-\mathrm{V}$ axis by numerous secreted factors. We have shown that many Gpcs and Notum, which have been proposed to regulate the activity of secreted factors, are also expressed in this tissue. To establish an overview of the impact of Notum on DV patterning we unilaterally electroporated the full length mouse version of the molecule into the right hand side of $\mathrm{HH} 10-12$ chick

embryo neural tube for a period of 16-24 h. The expression vector also expressed GFP that allowed us to monitor regions that had been manipulated. Robust GFP expression was seen in the right half of the neural tube (Fig. 10A and C). We determined whether the gene of interest was also expressed and performed in-situ to the mouse homologue of Notum.

To examine the effect of Notum over-expression on neural tube pattering we focused on key markers of the neural tube. Firstly, we examined the expression domains of Nkx-6.1 which is expressed by the undifferentiated ventral cells and Pax7 which is 
expressed by the dorsal cells. In mock electroporations, i.e. with vector containing only GFP, the DV expression levels of both Pax7 and Nkx-6.1 were the same on the manipulated side and the un-operated side (Fig. 10E and G). However, in embryos electroporated with mNotum, there was a statistically significant ventral shift of both Nkx-6.1 (7\%) and $\operatorname{Pax} 7$ (13.5\%) (Fig. 10F, H, K and M). In addition, we looked at the effect of mNotum over-expression on the Shh expression domain along the DV axis. IMAGE J analysis showed that the Shh domain decreased by $8.5 \%$ on the operated side (Fig. 10I, J, and M). Therefore, over-expression of Notum affected the dorsoventral patterning of the chick neural tube, with a shift of Pax7 and Nkx-6.1 domains to more ventral regions.

\section{Discussion}

The chick has been used as an experimental model to gain insights into vertebrate embryogenesis for a number of reasons including availability of embryos, its amenability to experimental approaches and clarity of in-situ based expression profiles generated with whole-mount techniques.

Data presented in this paper revealed that Gpcs and Notum were expressed in a stage and tissues-specific manner during chick embryogenesis. During the early developing stages (HH4-10) Gpc1, Gpc4, Gpc5 and Notum were expressed widely and strongly throughout the developing embryo while Gpc2 and Gpc3 were expressed in specific regions and Gpc6 displayed very weak expression.

The temporally early Gpcs and Notum expression suggests that these genes play important roles during gastrulation. As development progressed Gpcs and Notum expression became restricted to specific regions. For example, at stages $\mathrm{HH}-22$ and $\mathrm{HH}-24$ Gpc2, Gpc3, Gpc5 had become limited to specific tissues, suggesting that 
Gpcs may have important functions in the later embryonic developing stages. Gpcs and Notum may have a central role in the pattering events of embryonic structures, such as the neural tube.

Our data show that Gpc expression in specific tissues and structures was correlated with regions that express signalling molecules that are known to be regulated by Gpcs. Fgf4 in mammals and avian embryos is implicated in early developmental processes (Alvarez et al., 1998). Fgfs have important roles in the early developmental stages during mesoderm formation, anterior posterior patterning and neural tissue formation. Data from our current study show that Gpc1, Gpc4 Gpc5 and Notum were detected at $\mathrm{HH}-4$ in the primitive streak and the area around Hensen's node and this is consistent with an in-situ hybridisation study by Shamim et al., (1999) which showed that Fgf4 were first detected at $\mathrm{HH}-3$ in the anterior primitive streak and the expression become stronger in the central primitive streak and around Hensen's node (Shamim and Mason, 1999). It is possible that Gpc1, Gpc4 Gpc5 and Notum modulate the expression of Fgfs in these areas during the early developmental process.

We found that Gpc1, Gpc4, Gpc5 and Notum were expressed in the primitive streak and in the Hensen's node area at $\mathrm{HH}-4$ where Wnt-3a, Wnt-5a and Wnt-8c are known to be expressed (Hume and Dodd, 1993). Furthermore, Shh is expressed in Hensen's node with stronger expression on the left side. This structure also expresses Gpc1, Gpc4, Gpc5 and Notum. A number of studies have shown that Gpcs serve as co-receptors for Shh (Li et al., 2011). There seem to be two types of Gpc expression at early developmental stages; Gpc1,2,3 are expressed in entire cell populations within a given compartment whereas Gpc5 shows an intriguing punctate pattern within dorsal tissues. These results show the early ectoderm and epiblast is, 
at least at the molecular level a heterogeneous tissue. The properties imbued by Gpc5 expression in the early dorsal structures remains to be determined but it could be related to cell division as Gpc5 has been shown to promote proliferation. Therefore, the detailed expression profiles presented in this study imply that the Gpc/Notum axis has an important role to play in early chick development.

\section{Somite Patterning}

The patterning of the somites is a canonical example of how secreted signalling proteins control cell fate. Classical experiments have shown that newly formed somites are naïve in terms of fate and are instructed into developing dorsal dermis, body muscle or the vertebral column by a host of signals that originate from the floor plate, notochord (both secrete Shh), roof plate (Wnt and Bmps), dorsal ectoderm (Wnts) and lateral plate mesoderm (BMPs) (Schmidt et al., 2004; Wagner et al., 2000). The activities of these molecules are regulated by proteins that act as antagonists including Follistatin and Frizzled family members (Connolly et al., 1995; Lin et al., 2007). Here we show that Gpc and Notum activity also add complexity to the process of somite patterning.

One interesting aspect of Gpc expression in the somites is the robust transcription of three of the six Gpcs during the initial epithelialisation process (Gpc1, Gpc4 and Gpc5). At this stage there is low level Notum expression. The exact role of the Gpc expression in the young somites remains to be elucidated but one intriguing possibility is that it acts to dampen the potency of large numbers of patterning signals produced by neighbouring tissues. We suggest that the relatively small field of cells without such a mechanism would otherwise swamp the entire structure and be unable to translate into the precise orchestrated pattern that eventually emerges. 
Another interesting feature highlighted by the somite profiling is the discovery of patterns that do not segregate with cellular compartments. For example, the expression of Gpc4 is highest in regions immediately adjacent to the neural tube which would at a minimum cover areas of the sclerotome and the dermomyotome.

The expression of Gpcs and Notum have been poorly documented during early vertebrate embryogenesis. Our study gives a detailed profile of this axis during chick development and in addition highlights some fascinating differences between the expression of chick genes compared to their mouse counterparts. For example, we found that Gpc4 is robustly expressed during the early stages of chick development in particularly the hind brain and somites. However this is not the same for the mouse homologue where Yboy-Gonzalez and others have shown a much more restricted profile (Ybot-Gonzalez et al., 2005). mGpc4 appears not only quantitatively different to its chick counterpart but also qualitatively unique in that the chick gene is expressed at sites where the mouse version is not, for example in the dorsal limb mesenchyme. It could be that the qualitative differences could be covered by other family members in the mouse. Detailed expression profiling in mammalian tissues could settle this matter. Most importantly these results suggest that the Gpc/Notum axis has an important role in vertebrate somite development. Indeed a recent study showed a novel mechanism involving the patterning of somites involving neural crest through the action of Gpc4 (Serralbo and Marcelle, 2014).

\section{Neural Tube Patterning}

Chick Gpc and Notum showed a very interesting expression profile in the midline structures; the neural tube and the notochord. Our study showed that these structures expressed Gpc1, 3, 4 and 5 as well as Notum. The later was particularly 
interesting as it was expressed in the dorsal neural tube from early stage $\mathrm{HH}-10$ and the expression maintained until later stage $\mathrm{HH}-26$. In the notochord it was expressed from $\mathrm{HH}-10$ to $\mathrm{HH}-18$. This suggests a putative role for Gpcs in neural tube patterning. Several subclasses of neurons are generated at specific dorsal-ventral positions in the neural tube in response to gradient concentrations of Shh expressed by the floor plate. Precise Shh concentration defines the identity of ventral neural progenitor cells by expressing unique combinations of transcription factors (Ericson et al., 1997). In contrast the roof plate and other dorsal tissues secrete members of the BMP and Wnt family of polypeptides to dorsalise the fate of neural tube cells (Altmann and Brivanlou, 2001). Here we show that over-expression of Notum in the neural tube results in its dorsalisation; extension of the expression domain of the dorsal marker Pax7 and reduction in the expression domain of the ventral markers Nkx6.1 and Shh. Over-expression of Notum could change the neural tube landscape by either affecting dorsal or ventral signalling by modulating the action of Gpcs. A huge body of research has demonstrated Gpc modulation of BMP signalling, both in invertebrates and vertebrates (Grisaru et al., 2001; Jackson et al., 1997). Pertinent to this study was the finding by Dwivedi et al who showed that Gpcs inhibit BMP signalling (Dwivedi et al., 2013). Therefore one explanation of the dorsalised neural tube is that the inhibitory action of Gpcs (specifically Gpc4 and 5, based on expression profiles) on Bmps is lifted by over-expression of Notum.

Alternatively Notum over-expression could affect the ventralising activity of the floor plate. Again a large body of evidence exists showing that Gpcs promote Shh signalling (Li et al., 2011; Wilson and Stoeckli, 2013) and that Shh expression from the notochord induces the expression of Shh in the floor plate which then patterns the neural tube (Roelink et al., 1994). Therefore it is conceivable that the decrease 
in Shh expression we reported after Notum over-expression is due to a disruption of the Shh auto-regulatory cascade underpinned by the Gpcs.

In summary we present the first full detailed profile of Gpc and Notum expression during chick embryonic development. We show that these genes have unique temperal/spatial expression patterns. Some genes (e.g. Gpc1, Gpc4 and Notum) are expressed at high levels in multiple tissues during the development of the organism whereas others (especially Gpc6) are expressed at a few sites at low levels. The study highlights that signalling mechanisms that control all tissue patterning through the action of secreted proteins are going to be regulated by the action of the Gpc/Notum axis. 
Legends

Figure 1: Expression pattern of Gpc1-Gpc6 at $\mathrm{HH}-4$.

(A-F) Whole mount in situ hybridisation of Gpc1-Gpc6 respectively. Dotted lines indicated the transverses sections planes presented in $A^{\prime}-F^{\prime \prime \prime}\left(A^{\prime}, A^{\prime \prime}\right)$ Gpc1 expressed in the ectoderm and epiblast respectively (red arrow), (A") Gpc1 expression in the ingression mesoderm (red arrow head). (B') Gpc2 expressed in low level in the ingressing tissue (red arrow). (C') Gpc3 was expressed in level in epiblast (red arrow). (C", C"') Gpc3 expressed in epiblast. (D') Gpc4 was expressed in the ingressing cells at level of Hensen's Node (red arrow). (D") Gpc4 was expressed in the hypoblast (red arrowhead). (E') Punctate Gpc5 expression in the ectoderm (red arrow). (E'-E'”) Punctate Gpc5 expression in the epiblast (red arrowhead). (F') Gpc6 was expressed in very low level in the epiblast (red arrowhead). (F"'-F'”) No Gpc6 expression in posterior regions.

Figure 2: Expression pattern of Gpc1-Gpc6 at HH7-8.

(A-F) Whole mounts in-situ hybridisation embryos of Gpc1 HH-7, Gpc2 HH-7+, Gcp3 $\mathrm{HH}-7$, Gpc4 HH-8, Gpc $5 \mathrm{HH}-7$, Gpc6 HH-7 respectively. Section planes are indicated by black dotted lines. (A'-F'"). ( $\left.A^{\prime}\right)$ Gpc1 was expressed in the ectoderm (red arrow). (A') Gpc1 was expressed in Hensen's Node and ectoderm (red arrow). (B') Detail of anterior expression of Gpc2. (C') Gpc3 expression in the floor of pharynx (red arrow). (C') Gpc3 was expression in the ectoderm (neural) (red arrow). (D') Gpc4 expression in the head fold (red arrow). (D") Gpc4 was expressed in the ectoderm (red arrow) and endoderm and mesoderm (red arrow head). (E') Gpc5 
expression in anterior neural tube. (E”) Gpc5 expression in high level in the neural plate and ectoderm (red arrow). (F') Gpc6 expression in the floor of pharynx (red arrow). (F") Gpc6 expressed at very low level in the ectoderm (red arrow).

Figure 3: The expression pattern of Gpc1-Gpc6 at HH10-12.

(A-F) Whole mounts in-situ hybridisation embryos of Gpc1 HH-12, Gpc2 HH-12, Gcp3 HH-12, Gpc4 HH-11, Gpc $5 \mathrm{HH}-10$, Gpc6 HH-12 respectively. Section planes are indicated by black dotted lines (A'-F'").

(A') Transverse section in the hindbrain, Gpc1 was expressed in the otic placode (red arrow), and neural tube (red arrowhead), (A") Gpc1 expression in the dermomyotome (red arrow head), and notochord (red head). (A"') Gpc1 expression in the segmental plate (red arrow head) and notochord (red head). (B') Gpc2 expression in the neural tube (red arrow head), head mesenchyme (red arrow). (B") Gpc2 expression in the neural tube (red arrow), head mesenchyme (red arrow head), and ectodermal floor of pharynx (black arrow). (B'") Transverse section of differentiated somite depicting Gpc2 expression in the sclerotome (red arrow). (C') At hind brain level, Gpc3 expression in the in the anterior tip of foregut (red arrow). (C") Expression in the heart tube (red arrow). (C") Somites failed to express Gpc3. (C'”) No expression in posterior. (D') Gpc4 expression in the hind brain (red arrow) and in the head mesenchyme (red arrowhead), (D") Gpc4 expression in the neural tube (red arrow), in the thick walled floor of pharynx (red arrow head) and sclerotome (black arrow). (D'") Gpc4 expression in the roof plate and floor plate (red arrowhead and blue arrowhead) respectively and sclerotome (red arrow), in the lateral plate mesoderm. (E') Gpc5 expression in the neural tube (red arrow). (E') Gpc5 expression in the head mesenchyme (red arrow). (E"') Gpc5 expression in the 
epithelial somite (red arrow). (F'-F') Weak Gpc6 expression in the developing brain region (red arrow) but not in the spinal cord part of the neural tube (F'").

Figure 4: Expression pattern of Gpc1-Gpc6 during HH18-19.

(A-F) Whole mounts in-situ hybridisation embryos of Gpc1 $\mathrm{HH}-19$, Gpc2 $\mathrm{HH}-19$, Gcp3 HH-18, Gpc4 HH-19, Gpc5 HH-19, Gpc6 HH-19 respectively. Section planes are indicated by black dotted lines ( $\left.\mathrm{A}^{\prime}-\mathrm{F}^{\prime \prime \prime}\right)$.

(A) Gpc1 was expressed in the brain (blue arrow head) and in the fore and hind limb bud (red arrowheads). (A') Gpc1 expression in the roof plate (red arrow head) and dermomyotome (red arrow). (A') Gpc1 expression in the forelimb bud dorsal mesoderm (red arrowhead) and in the dorsal neural tube (blue arrowhead), as well as ( $\left.\mathrm{A}^{\prime \prime \prime}\right)$ in the hind limb bud ectoderm (red arrowhead). (B) Gpc2 was expressed strongly in the head and neck region (red arrow), (B') dorsal to floor plate (red arrowhead). (B') restricted expression of Gpc2 to region adjacent to floor plate (red arrowhead) and in fore limb ectoderm and mesenchyme (red arrow). (B'"') Posterior part devoid of Gpc2 expression. (C) Gpc3 expression in head placodes (red arrowhead) and limbs hind limb bud (blue arrows). (C') Weak Gpc3 expression in middle (D-V) neural tube (red arrowhead). (C") Strong Gpc3 expression in fore limb ectoderm and mesenchyme (blue arrow) and gut mesoderm (red arrowhead). (C'”) Strong Gpc3 expression in hind limb (red arrow). (D) Strong segmental expression of Gpc4 as well in branchial arches (red arrowhead). (D') Gpc4 expression in dermomyotome (red arrow) and myotome (blue arrow head). (D") Gpc4 expression in the dorsal neural tube (blue arrow), region of somite adjacent to neural tube (red arrow), intermediate mesoderm (green arrow) and proximal fore limb mesoderm (red arrowhead). (D'”) Gpc4 expression in hind limb mesenchyme (red arrow), intermediate mesoderm (green arrow) and throughout young somite (blue 
arrowhead). (E) Gpc5 expression in the fore and hind limb bud (red arrows). (E') Gpc5 expression in the forelimb bud mesenchyme (red arrow). (E') Very little expression at inter limb level. (E'”) Proximal Gpc5 expression in hind limb (red arrow). (F) Gpc6 expression only in the otic placode (red arrow). (F'-F'”') Little Gcp6 expression in body.

Figure 5: Expression pattern of Gpc1-Gpc6 at $\mathrm{HH}-22$.

(A-F) Whole mounts in-situ hybridisation embryos of Gpc1- Gpc6 at $\mathrm{HH}-22$ respectively. Section planes are indicated by black dotted lines ( $\left.A^{\prime}-F^{\prime \prime \prime}\right)$.

(A) Gpc1 was expressed in the head (red arrows), in the fore and hind limb bud (black arrows). (A') Weak Gpc1 expression in the neural tube (red arrowhead), DRG (blue arrowhead), and dermomyotome (red arrow). (A") Expression in the fore limb mesenchyme (blue arrow), DRG (blue arrowhead), dorsal neural tube (red arrowhead) and dermomyotome (red arrow). (A"') Expression of Gpc1 in the dorsal neural tube (red arrowhead), limb ectoderm (blue arrow), AER (red arrow) and ventral body ectoderm (blue arrowhead). (B) Gpc expression in head and occipital region (red arrows). (B' and B'"') Gpc2 expression immediately dorsal to floor plate (red arrowhead). (C) Gpc3 expressed in the head placodes (red arrowheads), fore and hind limb bud (black arrow head). (C') Gpc3 in the dorsal neural tube (red arrow head) and ventral mesoderm (red arrow). (C") Gpc3 expression in the dorsal neural tube (red arrowhead) and fore limb mesenchyme (red arrow). (C'”') Gpc3 expression in the dorsal neural tube (red arrowhead) and hind limb mesenchyme. (D) Gpc4 expression in somites (red arrows) and branchial arches (red arrowhead). (D') Gpc4 expression in the roof plate (red arrow head) and dermomyotome (red arrow), (D”) Gpc4 expression in the roof plate (red arrow head), dermomyotome (red arrow) and 
in the fore limb bud mesenchyme (blue arrow). (D'”) Gpc4 expression inn roof plate (red arrow head), dermomyotome (red arrow) and in the sub-ectodermal hind limb bud mesenchyme (blue arrow). (E) Gpc5 expression in limbs (red arrowheads). (E') Gpc5 expression in the DRG (red arrow), (E') Gpc5 was expression in the forelimb bud mesenchyme (red arrow) and DRG (red arrowhead). (E'”) Gpc5 expression in the DRG (red arrowhead) and hind limb mesenchyme (red arrow). (F) Gpc6 expression in the otic placode (red arrowhead). (F'-F'") Little Gcp6 expression in body.

Figure 6: Expression pattern of Gpc1-Gpc6 during HH 24-25.

(A-F) Whole mount in situ embryo for Gpc1-Gpc5 expression at HH24 and Gpc6 at $\mathrm{HH}-25$ respectively. (A) Gpc1 in the fore and hind limb bud (red arrows head). (A') Gpc1 expressed in the somites (blue arrow) and branchial arch region (red arrow). (B and B') Gpc2 expression in the branchial arches (red arrow) in the fore and hind limb buds (red arrowsheads). (C) Gpc3 expression in the somites (red arrow) and fore and hind limb buds (red arrow head). (D) Gpc4 expression in optics region (red arrow), posterior margin of fore limb (blue arrow) and strong in posterior somites (white arrow). (D') Detail of cervical region showing strong expression of Gpc4 in dorsal midline (black arrow), somites (red arrow) and branchial arch (blue arrow). (E) Gpc5 expression in segmental pattern in the body (red arrowhead) and fore and hind limb (red arrow). (E') Detail of Gpc5 expression in segmental pattern in the cervical region. (F) Expression of Gpc6 in the heart at HH-25 (F') Detail showing expression ventral to somites in cervical region (red arrows).

Figure 7: Notum expression at $\mathrm{HH}-4-10$ 
(A) Dorsal view of HH-4 and (A') HH-7. Dotted line indicate section plane for B-D and B'-D'. (A) Notum expression along the primitive streak and adjacent to the Hensen's Node (red arrow). (B) Notum expression in ectoderm (red arrowhead). (C-D) Notum expression in epiblast with weak expression in ingressing cells (red arrowhead). ( $A^{\prime}$ ) Segmental paraxial expression of Notum. (B') Notum expression in the neural plate (red arrowhead) in head mesenchyme (blue arrowhead). (C') Notum expression indicated pre-somitic mesoderm (red arrow), neural plate (red arrowhead) and notochord (blue arrow). (D') Expression of Notum epiblast (red arrowhead) and ingressing cells (red arrow).

\section{Figure 8: Expression of Notum at $\mathrm{HH}-10-13$}

(A) Dorsal view of $\mathrm{HH}-10$ and $\left(\mathrm{A}^{\prime}\right) \mathrm{HH}-13$ embryo. Dotted line indicate section plane for B-D and B'-D'. (A) Notum expressed robustly in the head, somites and midline structures (red arrow). (B) Expression of Notum in the neural tube (red arrowhead) and head mesenchyme (red arrow). (C) Expression of Notum in dorsal neural tube (red arrowhead), throughout early differentiating somite (red arrow) and notochord (blue arrow). (C) Expression of Notum in the notochord (blue arrow) and pre-somitic mesoderm (red arrow). (A') Notum robustly expressed in the somites and midline structure (red arrow). (B') Expression of Notum in the dermomyotome (red arrowhead and lower levels in sclerotome (red arrow). Strong expression in notochord (blue arrowhead). (C') Weak Notum expression in dorsal neural tube (red arrowhead). Strong expression of Notum in dorsal region of epithelial somite (red arrow) and notochord (blue arrowhead). (D') Notum expression in neural tube (red arrowhead), notochord (blue arrowhead) and pre-somitic mesoderm (red arrow). 


\section{Figure 9: Notum expression $\mathrm{HH}-18$ to $\mathrm{HH}-26$}

(A) Dorsal view of HH-18 (A') HH-22 and (A") HH-26 embryo. Dotted line indicate section plane for B-D and B'-D'. (A) Expression of Notum in the branchial arches, somites, fore and hind limb buds. (B) Expression in in dorsal neural tube (red arrowhead) and dermomyotome (red arrow). (C) Fore limb expression (blue arrow), dermomyotome (red arrow), dorsal neural tube (red arrowhead) ad notochord (blue arrowhead). (D) Hind limb expression (red arrow), dermomyotome (red arrow), dorsal neural tube (red arrowhead) ad notochord (blue arrowhead). (A') Expression of Notum in the branchial arches (blue arrow), dorsal structure (red arrow), fore limb (blue arrowhead) and hypaxial somatic region (white arrow). (B') Notum in the dermomyotome (red arrow), dorsal neural tube (red arrowhead) and neural crest derived cells (blue arrowhead). (C') Expression in the fore limb dorsal ectoderm and AER (red arrow). (D') Expression in the hind limb dorsal ectoderm and AER (red arrowhead). (A") Robust expression of Notum in body segments and limbs. (B") Expression of Notum in epaxial lip (red arrow), around the DRG (blue arrow) and in dorsal neural tube (red arrowhead). (C") Expression in the dermomyotome (red arrow) and around DRG (red arrowhead). (D") Expression in dermal sub-ectodermal mesenchyme (red arrow) and AER (red arrowhead).

\section{Figure 10: Notum overexpression in the neural tube}

(A) Dorsal view chick embryo electroporated at $\mathrm{HH}-11$ and viewed 16h later for showed the GFP expression in the neural tube. (B) Whole mount in-situ hybridisation of embryo electroporated with mouse Notum (red arrow). (C) Transverse section in the electroporated area showing unilateral GFP expression. (D) Transvers section in the electroporated embryo showing mNotum expression in the neural tube. (E) 
Whole mount for Nkx6.1 and Pax7 in control electroporated embryo. (G) No change in expression level of Nkx6.1 (blue arrow) or Pax7 (red arrow) in control embryo. Red arrows indicates Pax7 expression and blue Nkx6.1. Note ventral shift of both in $(\mathrm{H})$. (F) Effect of Notum over-expression on Pax7 and Nkx-6.1 expression. (H). Section of mNotum over-expression embryo. Red arrows indicates Pax7 expression and blue Nkx6.1. Note ventral shift of both markers. (I) Wholemount for Shh in mNotum electroporated embryo. (J) Section of mNotum over-expression and Shh expression Red arrows indicate Shh expression Note ventral shift in Shh on right side. (K-M) Dorsal-ventral expression domains (\% length) in control and mNotum electroporated embryos of (K) Nkx6.1, (L) Pax7 and (M) Shh. Error bars in graphs represent standard error. 3 embryos per treatment were analysed. Asterisk denotes statistical significance where $p<0.05$.

\section{References}

Altmann, C.R., and Brivanlou, A.H. (2001). Neural patterning in the vertebrate embryo. Int Rev Cytol 203, 447-482.

Alvarez, I.S., Araujo, M., and Nieto, M.A. (1998). Neural induction in whole chick embryo cultures by FGF. Dev Biol 199, 42-54.

Bernfield, M., Gotte, M., Park, P.W., Reizes, O., Fitzgerald, M.L., Lincecum, J., and Zako, M. (1999).

Functions of cell surface heparan sulfate proteoglycans. Annu Rev Biochem 68, 729-777.

Campos-Xavier, A.B., Martinet, D., Bateman, J., Belluoccio, D., Rowley, L., Tan, T.Y., Baxova, A., Gustavson, K.H., Borochowitz, Z.U., Innes, A.M., et al. (2009). Mutations in the heparan-sulfate proteoglycan glypican 6 (GPC6) impair endochondral ossification and cause recessive omodysplasia. Am J Hum Genet 84, 760-770.

Capurro, M., Martin, T., Shi, W., and Filmus, J. (2014). Glypican-3 binds to Frizzled and plays a direct role in the stimulation of canonical Wnt signaling. J Cell Sci 127, 1565-1575.

Capurro, M.I., Xu, P., Shi, W., Li, F., Jia, A., and Filmus, J. (2008). Glypican-3 inhibits Hedgehog signaling during development by competing with patched for Hedgehog binding. Dev Cell 14, 700711.

Connolly, D.J., Patel, K., Seleiro, E.A., Wilkinson, D.G., and Cooke, J. (1995). Cloning, sequencing, and expressional analysis of the chick homologue of follistatin. Dev Genet 17, 65-77.

Dwivedi, P.P., Grose, R.H., Filmus, J., Hii, C.S., Xian, C.J., Anderson, P.J., and Powell, B.C. (2013).

Regulation of bone morphogenetic protein signalling and cranial osteogenesis by Gpc1 and Gpc3. Bone 55, 367-376.

Ericson, J., Rashbass, P., Schedl, A., Brenner-Morton, S., Kawakami, A., van Heyningen, V., Jessell, T.M., and Briscoe, J. (1997). Pax6 controls progenitor cell identity and neuronal fate in response to graded Shh signaling. Cell 90, 169-180. 
Esko, J.D., Kimata, K., and Lindahl, U. (2009). Proteoglycans and Sulfated Glycosaminoglycans.

Filmus, J., and Capurro, M. (2008). The role of glypican-3 in the regulation of body size and cancer. Cell Cycle 7, 2787-2790.

Filmus, J., Capurro, M., and Rast, J. (2008). Glypicans. Genome Biol 9, 224.

Filmus, J., and Selleck, S.B. (2001). Glypicans: proteoglycans with a surprise. J Clin Invest 108, 497501.

Grisaru, S., Cano-Gauci, D., Tee, J., Filmus, J., and Rosenblum, N.D. (2001). Glypican-3 modulates BMP- and FGF-mediated effects during renal branching morphogenesis. Dev Biol 231, 31-46.

Hamburger, V., and Hamilton, H.L. (1951). A series of normal stages in the development of the chick embryo. J Morphol 88, 49-92.

Hume, C.R., and Dodd, J. (1993). Cwnt-8C: a novel Wnt gene with a potential role in primitive streak formation and hindbrain organization. Development 119, 1147-1160.

Jackson, S.M., Nakato, H., Sugiura, M., Jannuzi, A., Oakes, R., Kaluza, V., Golden, C., and Selleck, S.B. (1997). dally, a Drosophila glypican, controls cellular responses to the TGF-beta-related morphogen, Dpp. Development 124, 4113-4120.

Khare, N., and Baumgartner, S. (2000). Dally-like protein, a new Drosophila glypican with expression overlapping with wingless. Mech Dev 99, 199-202.

Khare, N., Fascetti, N., DaRocha, S., Chiquet-Ehrismann, R., and Baumgartner, S. (2000). Expression patterns of two new members of the Semaphorin family in Drosophila suggest early functions during embryogenesis. Mech Dev 91, 393-397.

Kreuger, J., Perez, L., Giraldez, A.J., and Cohen, S.M. (2004). Opposing activities of Dally-like glypican at high and low levels of Wingless morphogen activity. Dev Cell 7, 503-512.

Li, F., Shi, W., Capurro, M., and Filmus, J. (2011). Glypican-5 stimulates rhabdomyosarcoma cell proliferation by activating Hedgehog signaling. J Cell Biol 192, 691-704.

Lin, C.T., Lin, Y.T., and Kuo, T.F. (2007). Investigation of mRNA expression for secreted frizzledrelated protein 2 (sFRP2) in chick embryos. J Reprod Dev 53, 801-810.

Lin, X. (2004). Functions of heparan sulfate proteoglycans in cell signaling during development. Development 131, 6009-6021.

Luxardi, G., Galli, A., Forlani, S., Lawson, K., Maina, F., and Dono, R. (2007). Glypicans are

differentially expressed during patterning and neurogenesis of early mouse brain. Biochem Biophys Res Commun 352, 55-60.

Nieto, M.A., Patel, K., and Wilkinson, D.G. (1996). In situ hybridization analysis of chick embryos in whole mount and tissue sections. Methods Cell Biol 51, 219-235.

$\mathrm{Niu}, \mathrm{S}$., Antin, P.B., Akimoto, K., and Morkin, E. (1996). Expression of avian glypican is developmentally regulated. Dev Dyn 207, 25-34.

Pei, J., and Grishin, N.V. (2012). Cysteine-rich domains related to Frizzled receptors and Hedgehoginteracting proteins. Protein Sci 21, 1172-1184.

Pilia, G., Hughes-Benzie, R.M., MacKenzie, A., Baybayan, P., Chen, E.Y., Huber, R., Neri, G., Cao, A., Forabosco, A., and Schlessinger, D. (1996). Mutations in GPC3, a glypican gene, cause the SimpsonGolabi-Behmel overgrowth syndrome. Nat Genet 12, 241-247.

Roelink, H., Augsburger, A., Heemskerk, J., Korzh, V., Norlin, S., Ruiz i Altaba, A., Tanabe, Y., Placzek, M., Edlund, T., Jessell, T.M., et al. (1994). Floor plate and motor neuron induction by vhh-1, a vertebrate homolog of hedgehog expressed by the notochord. Cell 76, 761-775.

Schmidt, C., Stoeckelhuber, M., McKinnell, I., Putz, R., Christ, B., and Patel, K. (2004). Wnt 6 regulates the epithelialisation process of the segmental plate mesoderm leading to somite formation. Dev Biol 271, 198-209.

Serralbo, O., and Marcelle, C. (2014). Migrating cells mediate long-range WNT signaling.

Development 141, 2057-2063.

Shamim, H., and Mason, I. (1999). Expression of Fgf4 during early development of the chick embryo. Mech Dev 85, 189-192. 
Song, H.H., and Filmus, J. (2002). The role of glypicans in mammalian development. Biochim Biophys Acta 1573, 241-246.

Veugelers, M., De Cat, B., Ceulemans, H., Bruystens, A.M., Coomans, C., Durr, J., Vermeesch, J., Marynen, P., and David, G. (1999). Glypican-6, a new member of the glypican family of cell surface heparan sulfate proteoglycans. J Biol Chem 274, 26968-26977.

Wagner, J., Schmidt, C., Nikowits, W., Jr., and Christ, B. (2000). Compartmentalization of the somite and myogenesis in chick embryos are influenced by wnt expression. Dev Biol 228, 86-94.

Wilson, N.H., and Stoeckli, E.T. (2013). Sonic hedgehog regulates its own receptor on postcrossing commissural axons in a glypican1-dependent manner. Neuron 79, 478-491.

Ybot-Gonzalez, P., Copp, A.J., and Greene, N.D. (2005). Expression pattern of glypican-4 suggests multiple roles during mouse development. Dev Dyn 233, 1013-1017. 


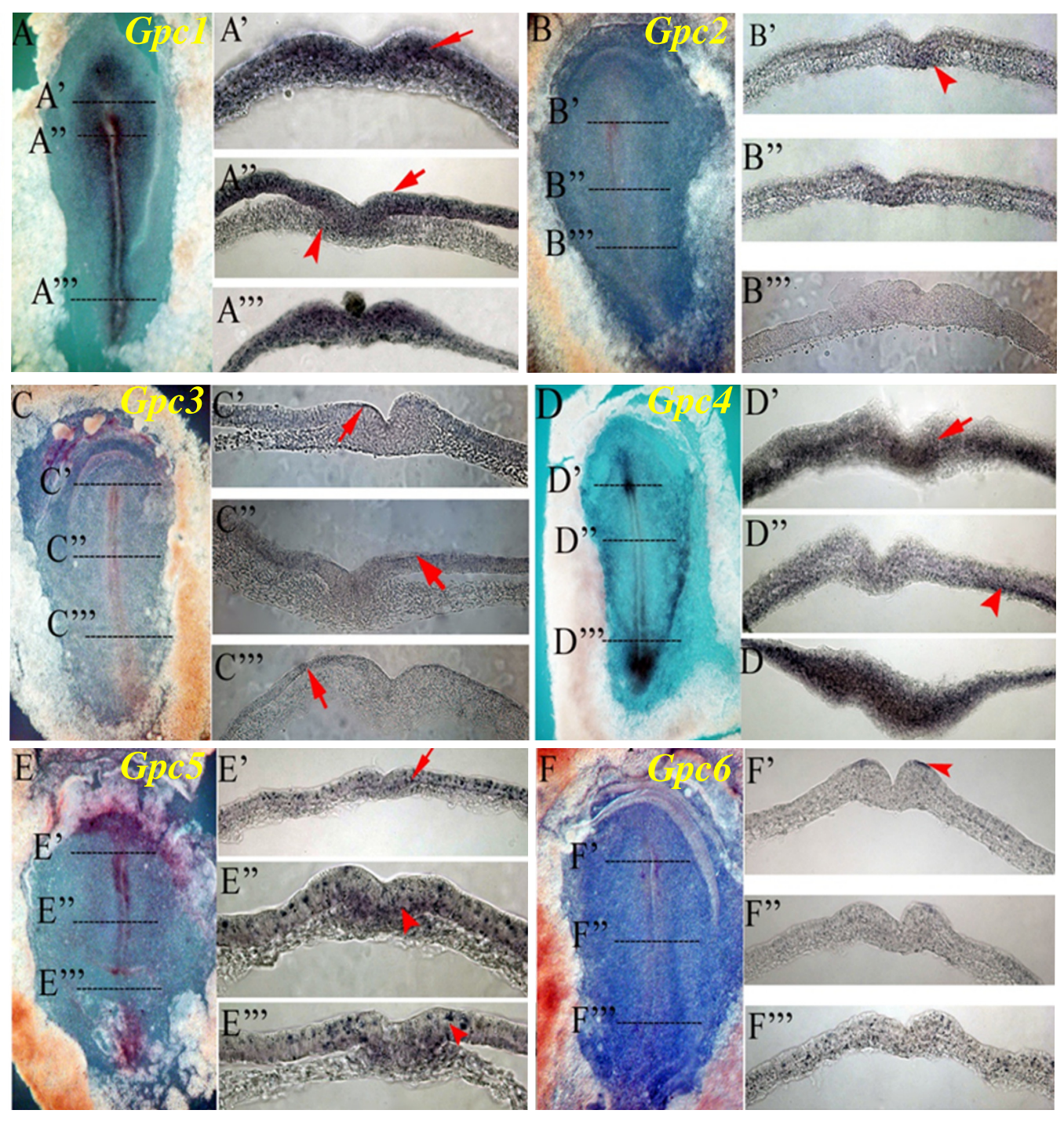

Fig 1 


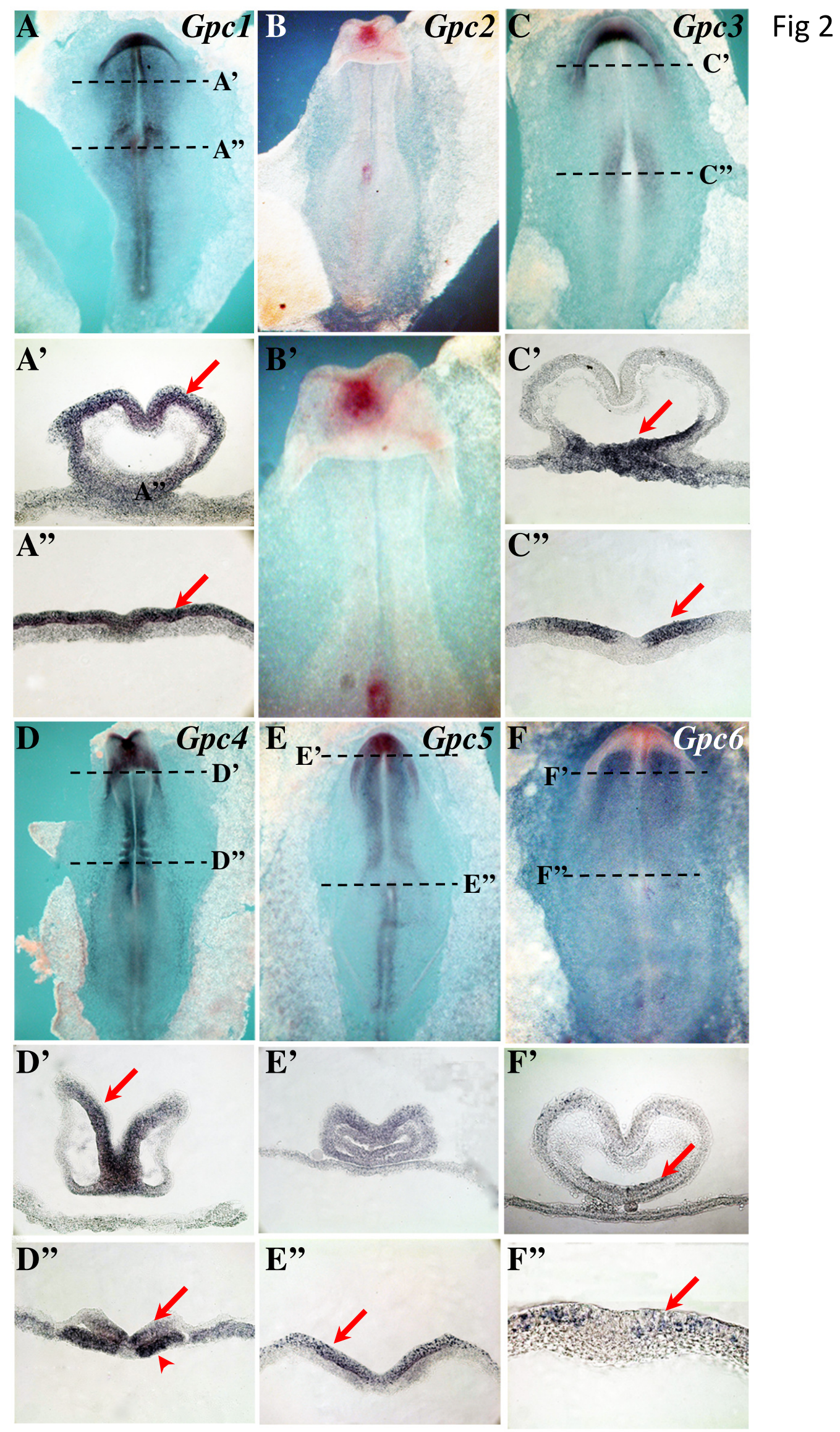


Fig 3
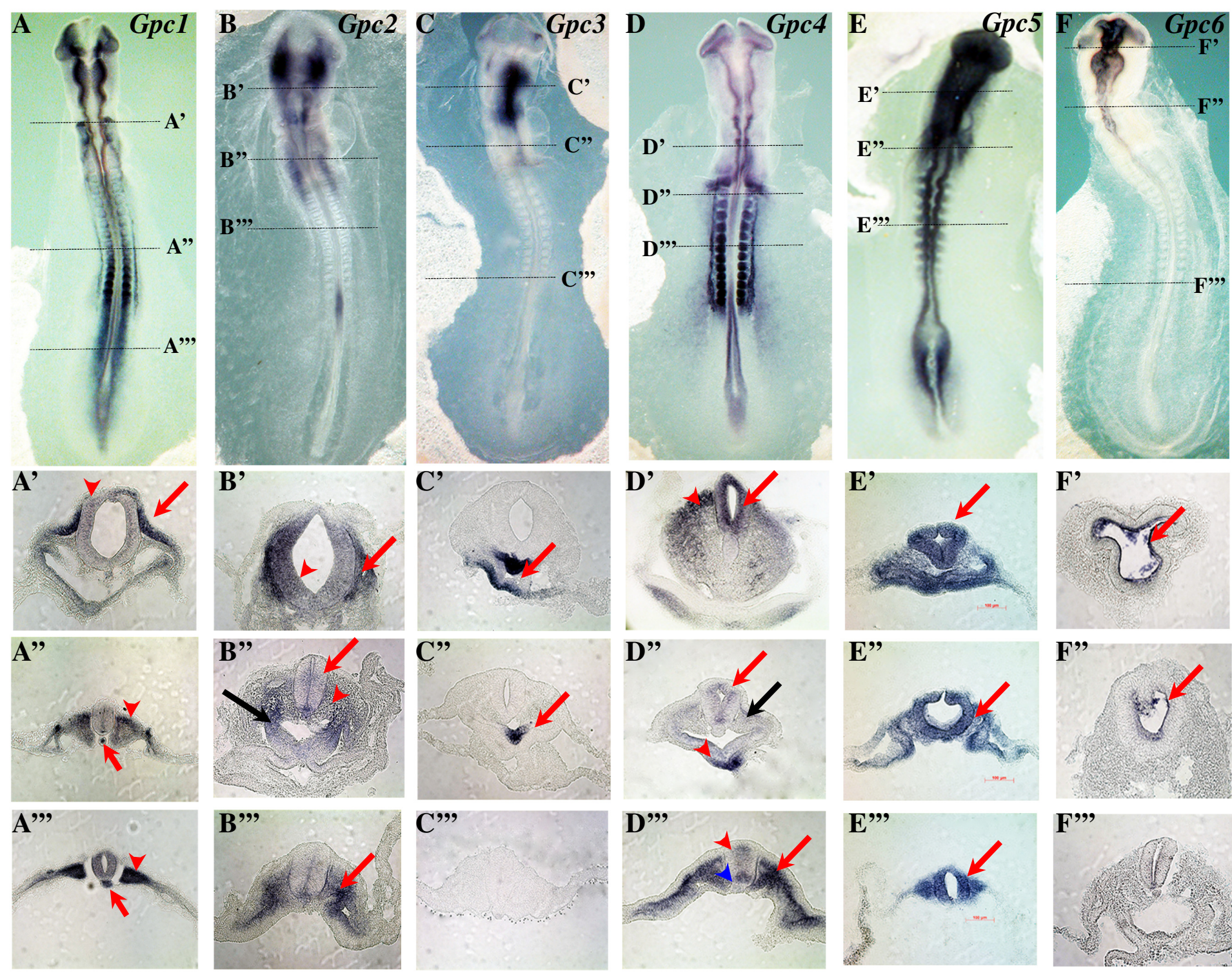


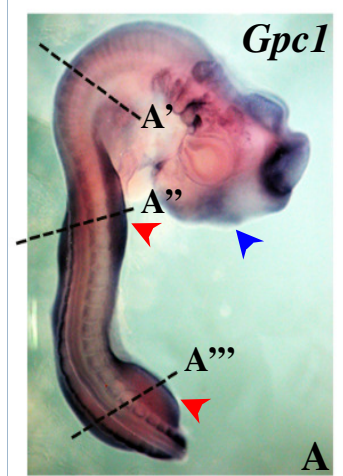

A
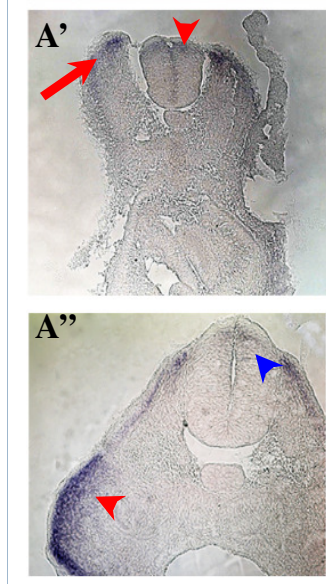

A"
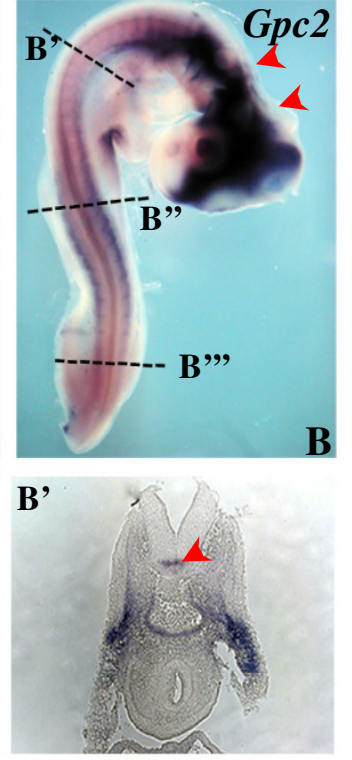

B” $\quad \nabla \quad$ C"

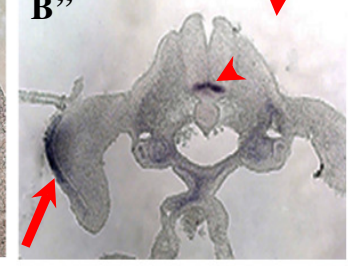

B"”

C'
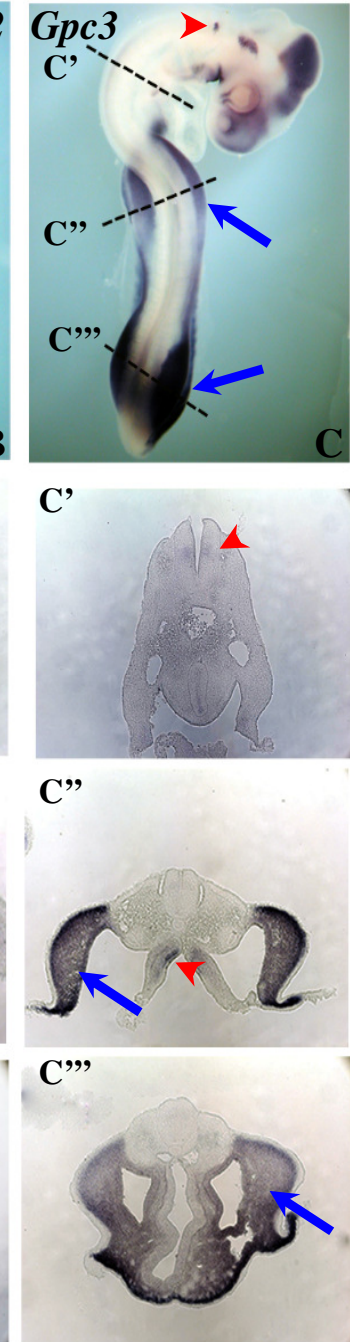

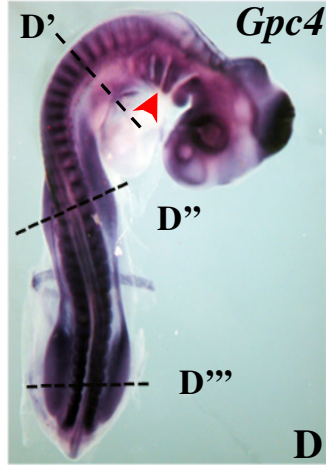

D

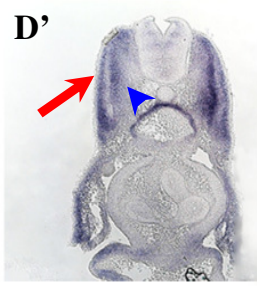

D”
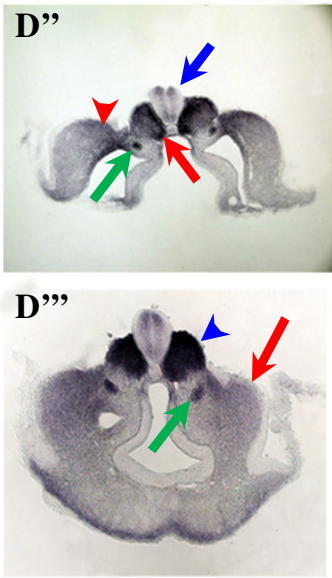
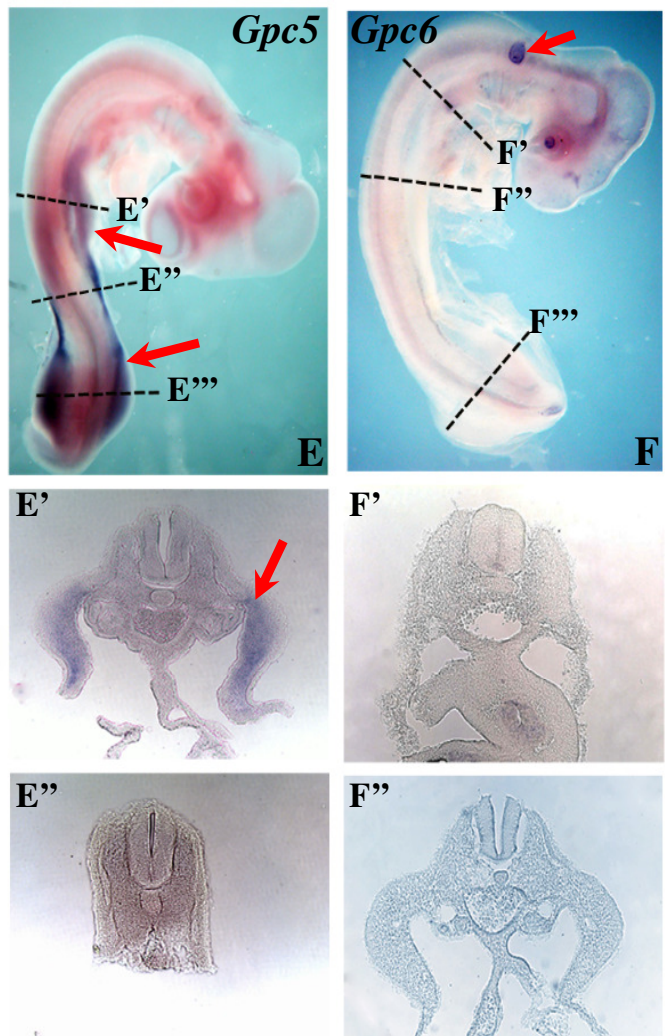

E"

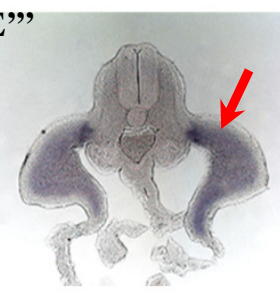

F'

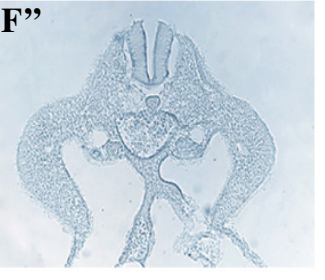

F"'

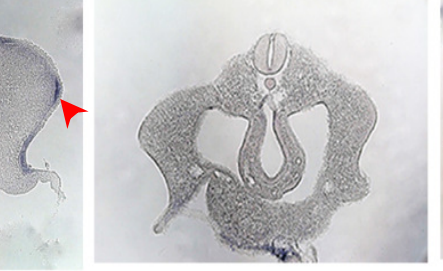

Fig 4 


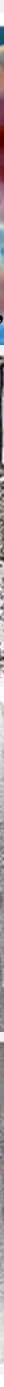

Fig 5 

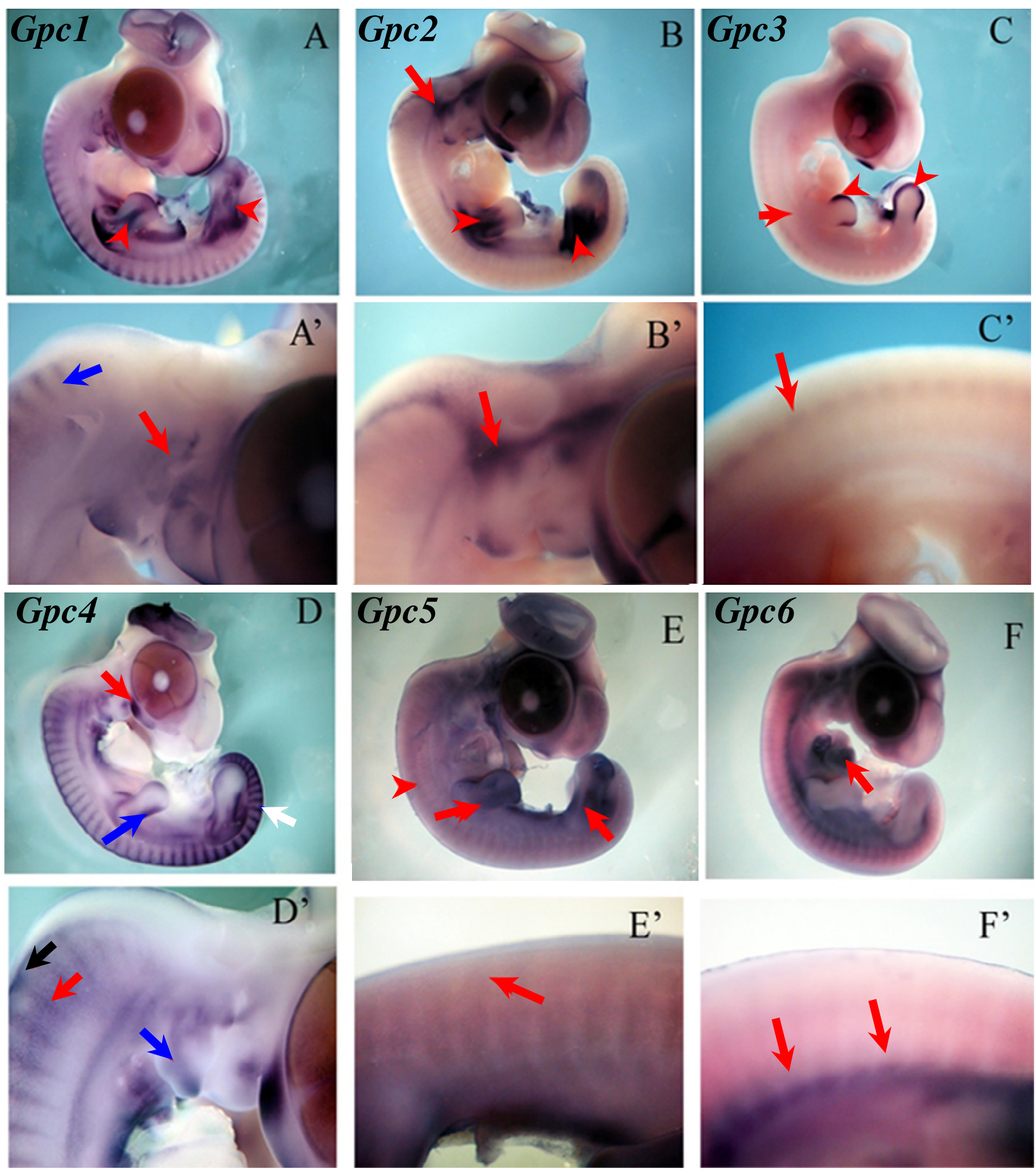

F'

Fig 6 


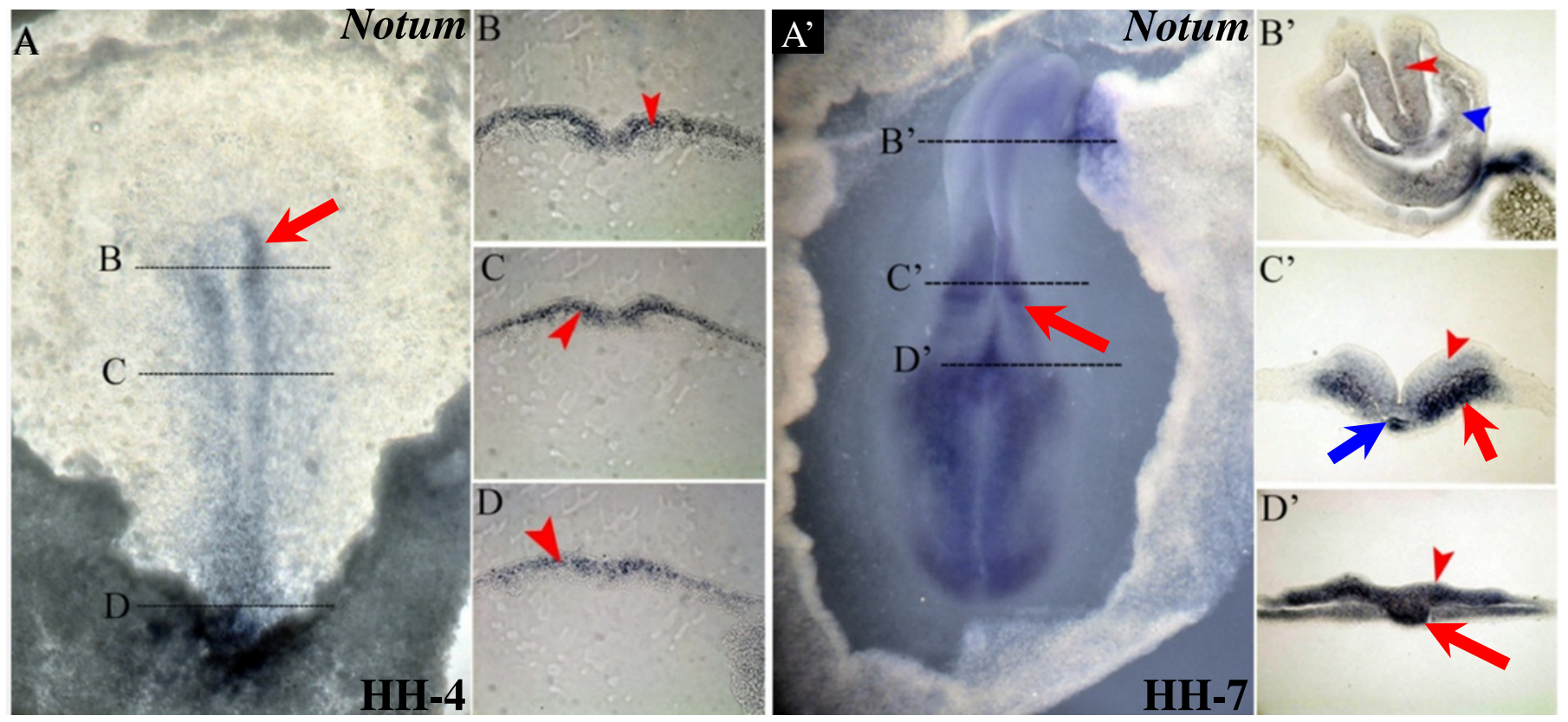

Fig 7 


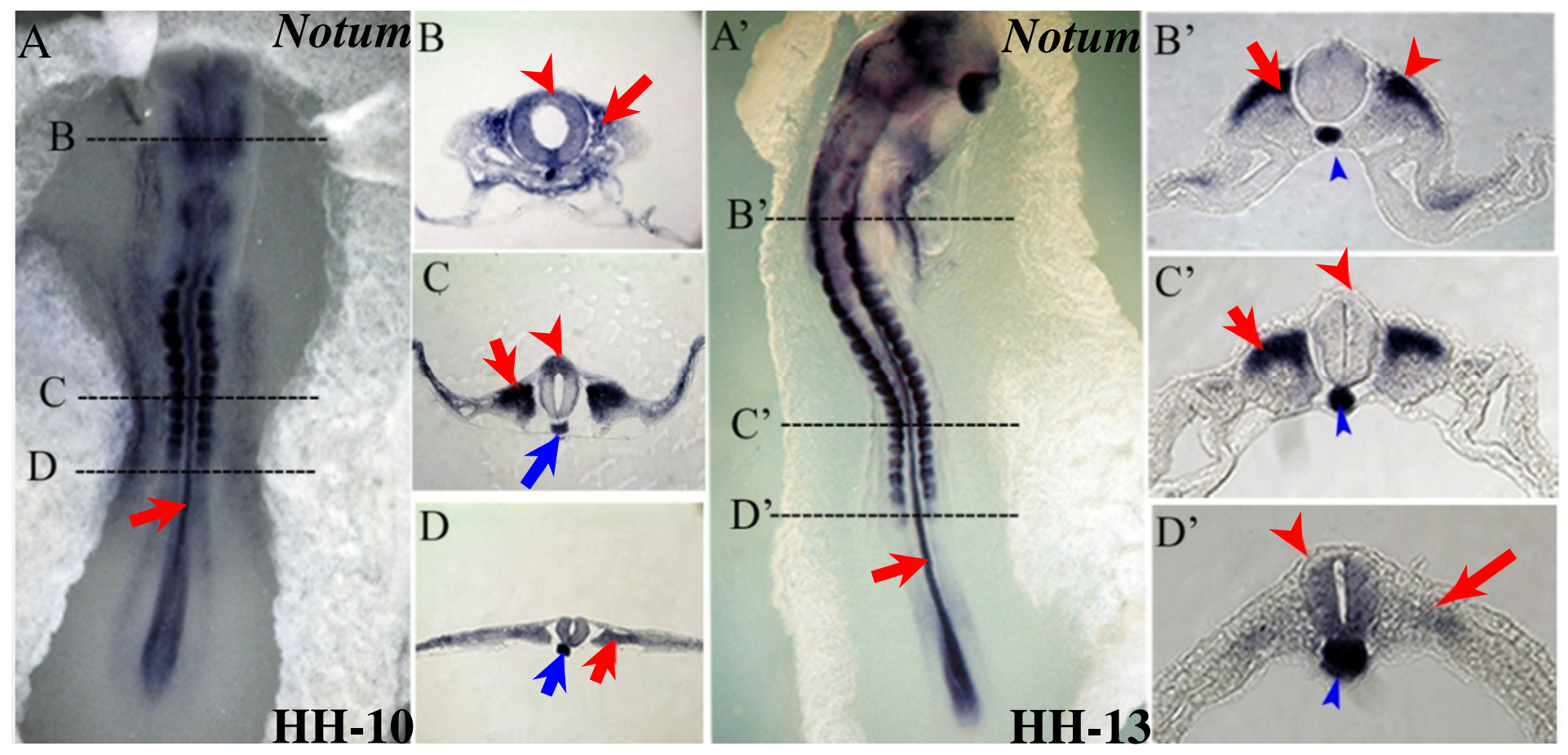

Fig 8 

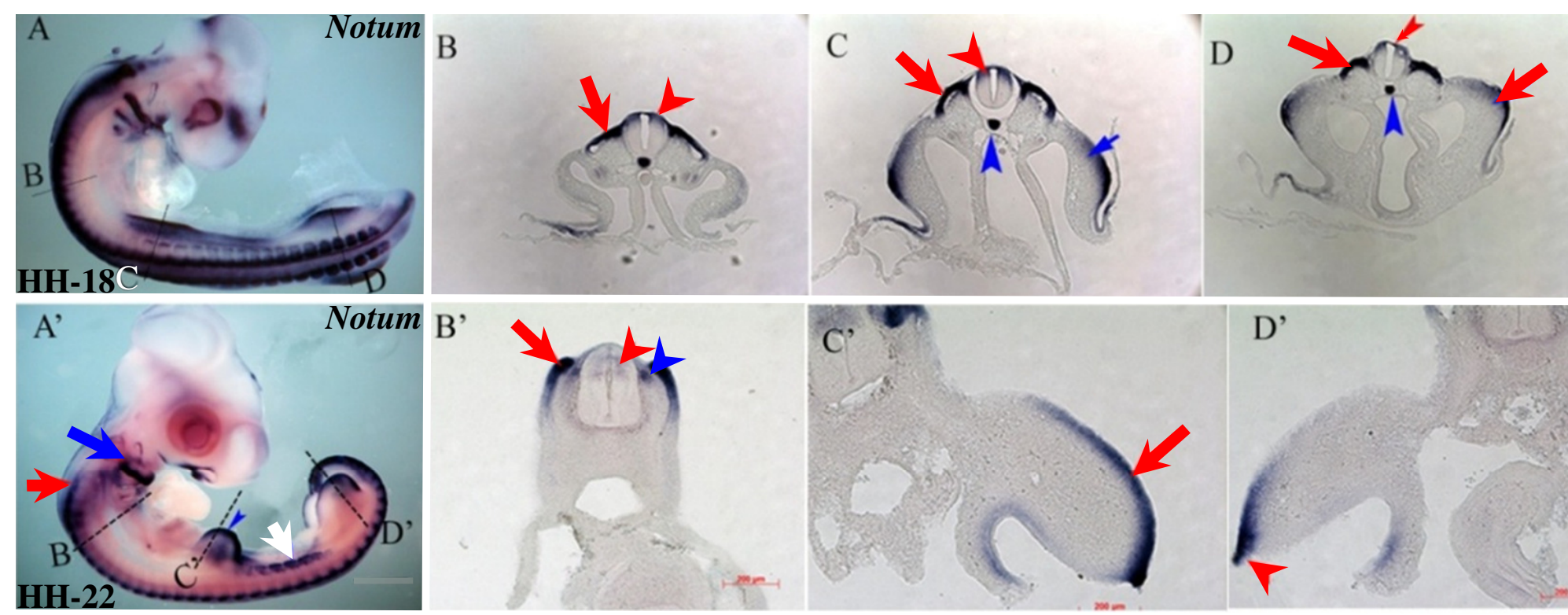

D'

A
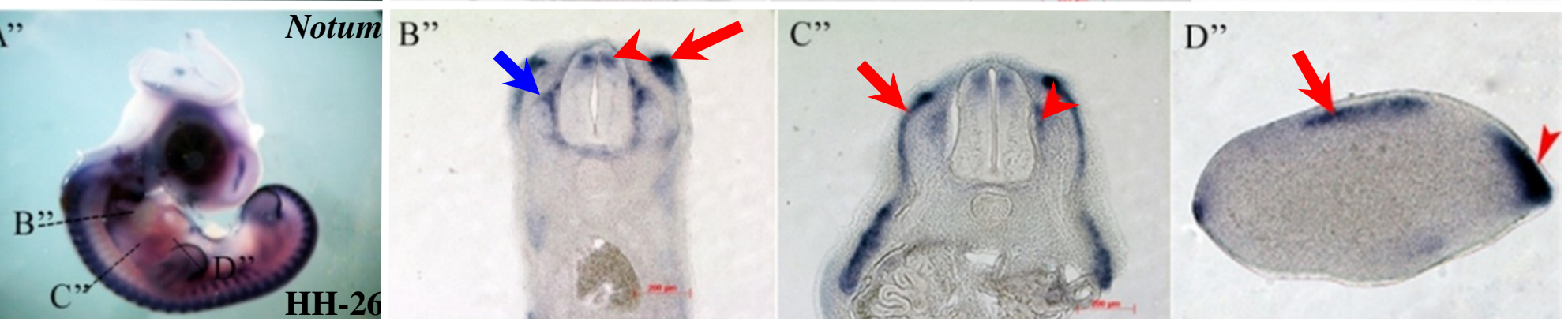

Fig 9 

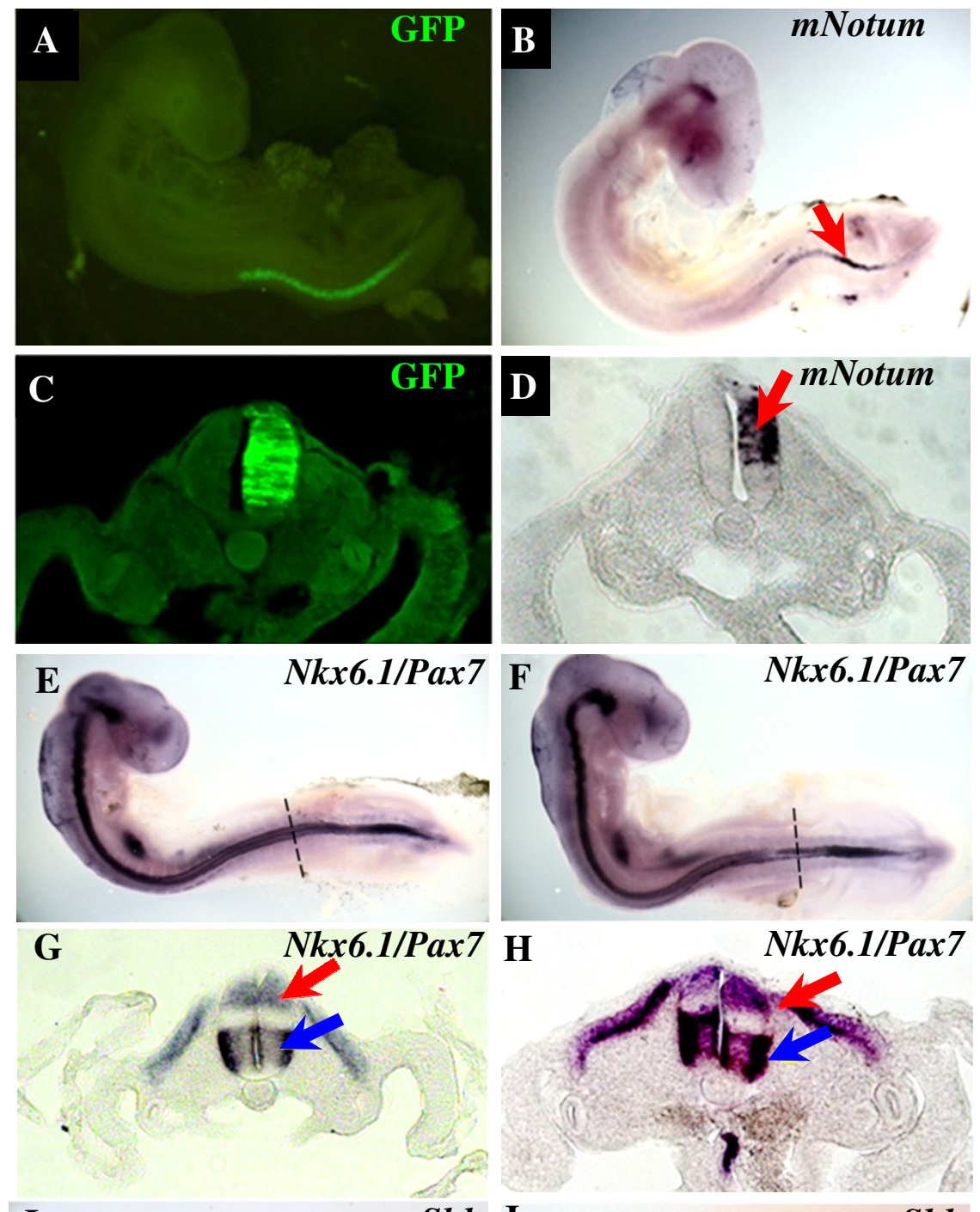

Nkx6.1/Pax 7

I

Shh J

Shh
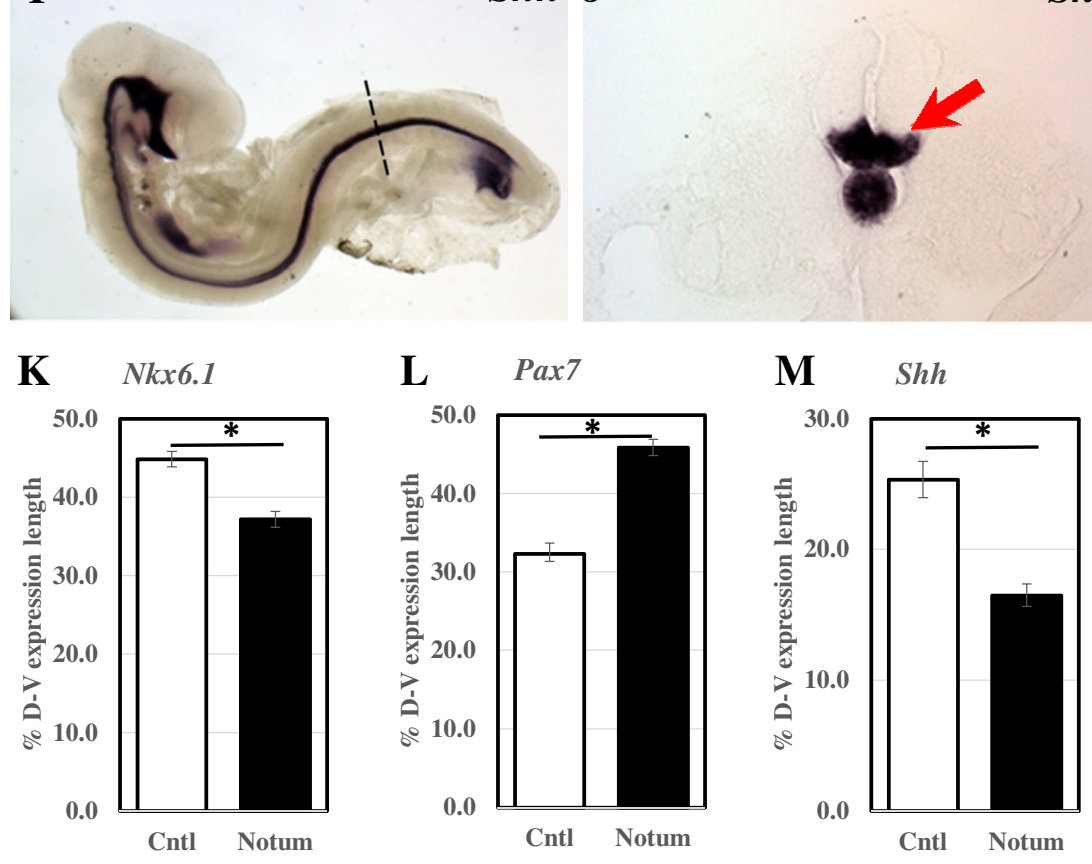

Fig 10 